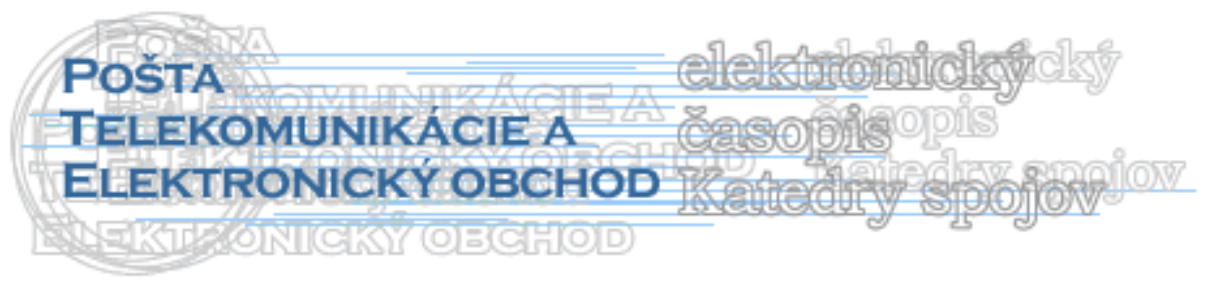

\title{
KUNDENZUFRIEDENHEIT DURCH QUALITÄTSMANAGEMENTSYSTEME
}

\author{
Thomas Bader, Iveta Kremeňová*
}

\section{Ausgangssituation}

Hohe Marktsättigung, harter Verdrängungswettbewerb durch die zunehmende Globalisierung der Märkte und immer kürzere Vermarktungszyklen beeinflussen heutzutage nahezu alle Unternehemensbranchen.

Daneben kommen, zu diesen allgemeinwirtschaftlichen Rahmenbedingungen, auch noch die hohe Transparenz der Märkte und die Möglichkeit der einfachen Informationsbeschaffung hinzu. Dies alles führt letztlich dazu, dass sich die Kunden immer kritischer und weniger loyal gegenüber den Unternehmen verhalten.

Waren in den Jahren zuvor sich die Unternehmensleitungen noch einig darüber, dass allein die Produktqualität der wichtigste Erfolgsfaktor ist, so steht heute neben Kundenbindung und Kundenorientierung, die Kundenzufriedenheit als der zentrale, wettbewerbsdifferenzierende Erfolgsfaktor an oberster Stelle.

Kundenzufriedenheit bildet somit die Basis des langfristigen Geschäftserfolges, und nur wenn es den Unternehmen gelingt, neben der Produkt- und / oder Dienstleistungsqualität auch einen hohen Grad an Kundenzufriedenheit zu erreichen, werden sie weiterhin am Markt existieren können.

\section{Kundenzufriedenheit durch Qualitätsmanagementsysteme}

Zufriedene Kunden sind die Grundpfeiler eines Unternehmens, denn diese Kunden kommen und kaufen wieder. Kundenzufriedenheit ist damit eine wesentliche Voraussetzung zur Existenzsicherung des Unternehmens.

Auf Grund dieser Erkenntnis entschließen sich immer mehr Unternehmen Qualitätsmanagementsysteme (QM-Systeme) einzuführen, verbunden mit den Erwartungen, dadurch neben der vom Markt geforderten hohen Qualität der erzeugten Güter, auch ein hohes $\mathrm{Ma}$ an Kundenzufriedenheit zu erreichen. Denn nur wer diesen Spagat schafft, wird dauerhaft erfolgreich sein.

Doch was versteht man unter dem Begriff „QM-System“ - nachfolgend eine Erklärung dazu:

\footnotetext{
* Thomas Bader, FIDUCIA, Regionaldirektion Südwest, Karlsruhe, NSR, Iveta Kremeňová, University of Zilina, Department of Communications, FPEDAS, tel.: +421 415133 100. 101, fax: +421 5655615

e-mail: Iveta.Krmenova@fpedas.uniza.sk
} 
Ein QM-System versteht sich als ein Verfahren / eine Arbeitsweise, für die Festlegung der Qualitätspolitik und von Qualitätszielen sowie dem Erreichen dieser Ziele. Dies ist die Gesamtheit der aufbau- und ablauforganisatorischen Gestaltung, sowohl zur Verknüpfung der qualitätsbezogenen Aktivitäten untereinander wie auch im Hinblick auf eine einheitliche, gezielte Planung, Umsetzung und Steuerung der Maßnahmen des Qualitätsmanagements im Unternehmen. Dabei wird nicht nur die Produktion mit ihren vor- und nachgelagerten Bereichen einbezogen, sondern das gesamte Unternehmen einschließlich der Beziehungen zu seinem Umfeld. Es entsteht ein systemvernetzter Regelkreis auf allen betrieblichen Ebenen, wodurch Ziele, Struktur, Verantwortlichkeiten, Verfahren, Prozesse und die zur Durchführung erforderlichen Mittel festgelegt werden. Das Qualitätsmanagementsystem dient somit der Strukturierung und der systematischen Umsetzung von Qualitätsaufgaben im Unternehmen [1].

Dass Kundenzufriedenheit ein bzw. der wesentliche Erfolgsfaktor (neben hoher Produktqualität) ist, untermauert beispielsweise die folgende aktuelle Umfrage aus dem Excellence-Barometer (ExBa).

Das ExBa ist die umfassendste Studie zur Leistungsfähigkeit der deutschen Wirtschaft und wird jährlich von der Mainzer forum! Markforschung $\mathrm{GmbH}$ und der Deutschen Gesellschaft für Qualität e. V. (DGQ) erhoben.

Demnach hängt der Erfolg bei deutschen Unternehmen entscheidend von der Kundenzufriedenheit und Kundenbindung, sowie von der Qualität der gelieferten Produkte und Dienstleistungen ab. Denn ein Drittel der 353 befragten Entscheider der ersten und zweiten Eben, hatten mit der Kundenzufriedenheit und Kundenbindung und ein Viertel mit der Qualität der Produkte und Dienstleistungen als die wichtigsten Kriterien für ihren Unternehmenserfolg genannt.

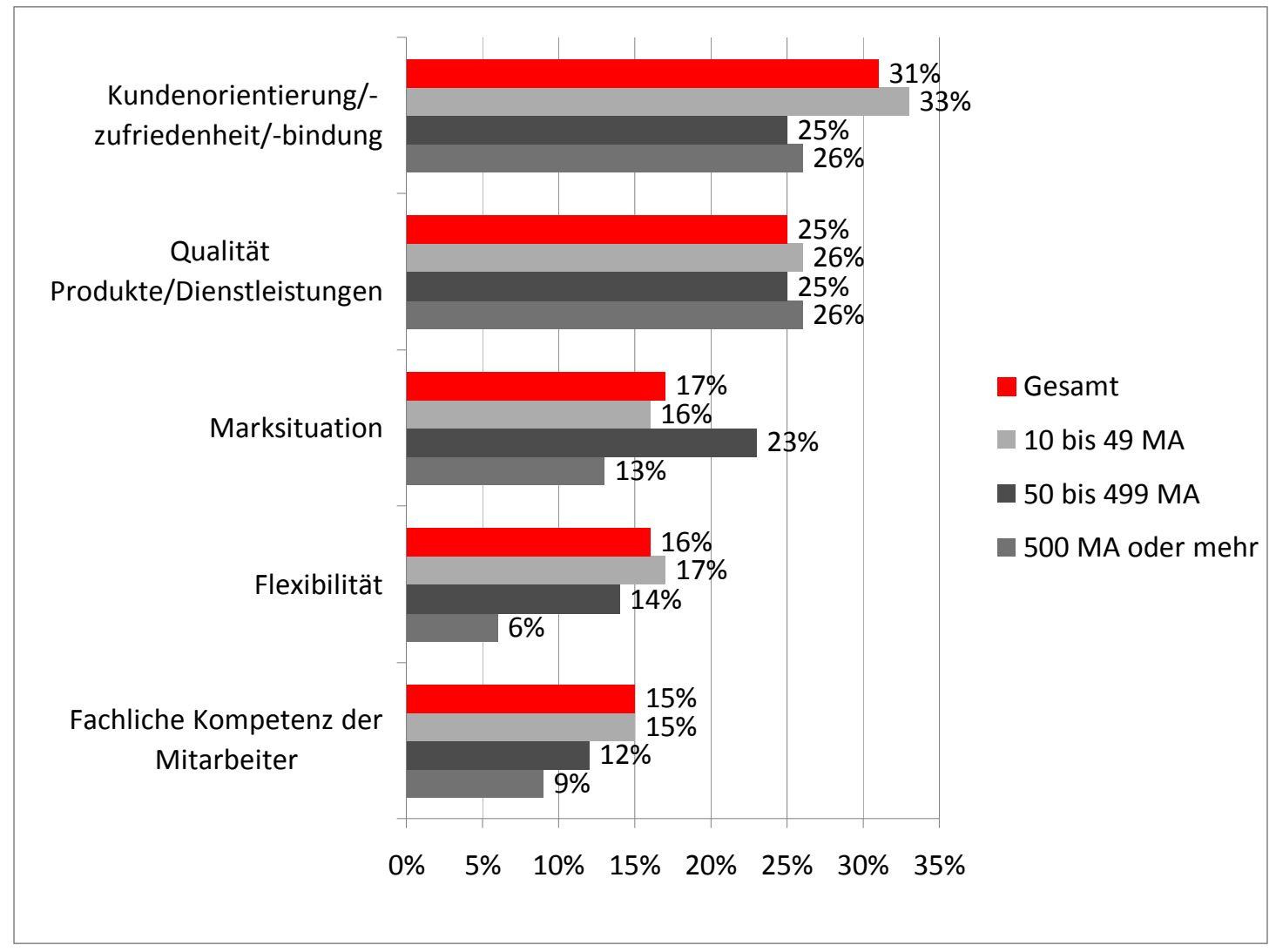


Das produzierende Gewerbe begründete den Unternehmenserfolg mit der Qualität seiner Produkte (28 Prozent). Demgegenüber konzentrierte sich der Handel (33 Prozent) und der Dienstleistungssektor (30 Prozent) in erster Linie auf die Zufriedenheit ihrer Kunden.

Künftig sollen zufriedene Kunden noch stärker an die Unternehmen gebunden werden. Dieses Ziel rückt bei zahlreichen Unternehmen immer mehr in den Mittelpunkt. So hat im Vergleich zum Jahr 2006 (20 Prozent) die Rolle der Kundenzufriedenheit und Kundenbindung im Jahre 2007 (31 Prozent) deutlich an Bedeutung gewonnen.

Ebenso ist die Qualität der Produkte und Dienstleistungen in diesem Zeitraum auf der Bewertungsskala der Befragten von 21 auf 25 Prozent gestiegen.

Weniger Bedeutung als noch vor einem Jahr (20 Prozent) wurde dagegen der allgemeinen Marktsituation beigemessen (17 Prozent). Gleichbleibend auf Vorjahresniveau folgen Flexibilität (16 Prozent) und die fachliche Kompetenz der Mitarbeiter (15 Prozent) als Voraussetzung für den Unternehmenserfolg [2].

Diese Ergebnisse der Studie von deutschen Unternehmen sind sicherlich ebenso auf viele andere Länder übertragbar, denn Kundenzufriedenheit ist ein Thema, das die gesamte Weltwirtschaft betrifft.

\section{- $\quad$ Integration von Kundenzufriedenheit in QM-Systeme}

Das Thema Kundenzufriedenheit ist mittlerweile wesentlicher Bestandteil von Qualitätsmanagementsystemen geworden. An dieser Entwicklung hatten neben der USA und den wichtigsten Industrieländern in Europa auch Japan einen entscheidenden Anteil.

Wissenschaftshistorisch betrachtet entstand diese Bewegung in den 50er und 60er Jahren, dort hatten japanische Firmen erhebliche Probleme auf den amerikanischen und europäischen Märkten zu bestehen.

Der japanischen Wirtschaft ist es aber gelungen mit einer Qualitätskampagne in den 70er und 80er Jahren des 20. Jahrhunderts eine hohe Markttransparenz zu erreichen. Dabei wurden durch intensive Bemühungen nicht nur die Qualität der Produkte in entscheidendem Maße verbessert, sondern auch Methoden im Hinblick auf eine ganzheitliche Qualitätsbetrachtung entwickelt und zur Anwendungsreife gebracht.

So entstand zum Beispiel das teilweise aus traditionellen Verhaltensweisen der Japaner sowie aus grundsätzlichen Überlegungen von W. E. Deming abgeleitet bezüglich der Anwendung in der japanischen Industrie bis in die 50er Jahre zurückverfolgbaren Bestrebungen der „ständigen Verbesserung““.

Zu Beginn des 20. Jahrhunderts war das Wort „Qualität“ kaum genutzt worden. Es wurde fast ausschließlich von „Güte“ gesprochen. In der Folgezeit gab es dann ein vollkommen heterogenes Verständnis von Qualität, das sich im Wesentlichen an dem Schlagwort „Kundenzufriedenheit“ orientierte.

Vor diesem Hintergrund entstanden dann bereichs- und unternehmensübergreifende Methoden, die über die reine Qualitätssicherung wesentlich hinausgingen. Diese eingeleitete Entwicklung führte dann mit Beginn der 90er Jahre des 20. Jahrhunderts zu einem umfassenden Qualitätsmanagement, was sich in Qualitätsmanagementsystemen niederschlug [3]. 


\section{- $\quad$ Gründe für die Einführung eines QM-Systems}

Da die Einführung eines QM-Systems zunächst einmal mit allerhand Aufwand und Kosten verbunden ist, muss es schon gute Gründe geben, damit sich ein Unternehmen zu diesem Schritt entschließt. Dabei können die Beweggründe von Unternehmen zu Unternehmen recht verschieden sein. Nachfolgend ein Überblick (auszugsweise) über die wichtigsten Gründe:

- Qualitätsverbesserung

- Qualitätssicherung

- Transparenz der Geschäftsprozesse

- Konkurrenzdruck

- Steigerung Kundenzufriedenheit

- Effizienzsteigerung

- Prozessoptimierung

- Kostensenkung

- Fehlerreduktion

- Erfüllen von Gesetzesauflagen

- Imageverbesserung

Sicherlich lassen sich noch weitere gute Gründe für die Einführung eines QM-Systems finden, doch an dieser Stelle soll dies einmal genügen.

Viel wichtiger ist es die Frage in diesem Zusammenhang $\mathrm{zu}$ beantworten, welche Nutzenstiftung ein QM-System für das Unternehmen bringt, und inwieweit dessen Erwartungen erfüllt werden.

\section{- $\quad$ Nutzenstiftung eines QM-Systems für das Unternehmen}

Um die Frage zu beantworten, welchen Nutzen ein Unternehmen durch die Einführung eines Qualitätsmanagementsystems hat, erkennt man Gründe sowohl innerhalb als auch außerhalb der Unternehmung.

Was den internen Bereich betrifft, so ist festzuhalten, dass Qualität nicht von alleine entsteht. Das Unternehmen möchte zwar, dass seine Leistungen Nutzen bringen und keine Fehler aufweisen. Als Beispiel sei hier die Automobilindustrie genannt, denn Autos dürfen keine kritischen Fehler zeigen und müssen dem Kunden die individuelle Beweglichkeit sicherstellen. Qualitätsmanagementsysteme bilden die notwendigen Voraussetzungen zur internen Erfüllung dieser Aufgaben und zur Erreichung dieser firmeninternen Ziele.

Qualitätsmanagementsysteme sind jedoch auch nach außen wirksam. Sie unterstützen die Zusammenarbeit mit den Lieferanten und den Kunden des Unternehmens, und schaffen bei Kunden und Lieferanten wie auch bei Gesetzgebern, Behörden und der Öffentlichkeit Vertrauen in die qualitative Leistung des Unternehmens. Dieses Vertrauen entsteht allmählich aus der Zusammenarbeit, wird aber durch dokumentierte Informationen und Nachweise vorbereitet. $\mathrm{Zu}$ diesem Zweck, wie auch zur internen Anwendung und Schulung, werden Qualitätsmanagementsysteme in einer zweckmäßigen Dokumentation beschrieben. Diese Dokumentation wird normalerweise hierarchisch aufgebaut in Form eines Handbuchs, in welchem die Qualitätspolitik, Qualitätsverfassung, die Aufbau- und Ablauforganisation sowie die qualitätsbezogenen Aufgaben und Zuständigkeiten in der gesamten Unternehmung behandelt werden. [4]. 
Das heißt, dass durch die Implementierung eines QM-Systems das Unternehmen in die Lage versetzt wird, dauerhaft überragende Produkt- und Service-Qualität anbieten zu können. Darüber hinaus führt das QM-System $\mathrm{zu}$ einer kontinuierlichen Verbesserung der Prozessqualität, zu Kostenreduzierungen und zur Erhöhung der Kundenzufriedenheit.

\section{- $\quad$ Einfluss und Auswirkungen von QM-Systemen auf wirtschaftliche Entwicklungen}

Die Praxis hat gezeigt, dass Qualitätsmanagementsysteme auch in einer freien Marktwirtschaft ihren Platz haben. Sie schreiben nicht die spezifischen Ausprägungsformen eines Führungssystems vor, sondern beschränken sich auf Gestaltungsregeln, nach welchen ein Führungssystem zu entwickeln, zu implementieren und zu betreiben ist.

Qualitätsmanagementsysteme sind keine Inseln, die isoliert betrachtet werden können, es handelt sich vielmehr um Systeme, deren Abläufe und Anforderungen Einfluss und Auswirkungen auf viele Bereiche von Wirtschaft, Unternehmen und Mitarbeiter haben.

Aus diesem Grunde bedarf es der genaueren Betrachtung um das Gesamtsystem und die gesamtwirtschaftlichen Zusammenhänge zu verstehen. Nachfolgend erfolgt eine nähere Betrachtung der wesentlichen Einflussbereiche.

\section{$\underline{\text { Die Weltwirtschaft }}$}

Auf Grund dessen, das die Globalisierung weiter fortschreitet und der internationale Handel prosperiert, wird nach Regularien gesucht, die den Käufern und Verkäufern mehr Sicherheit bieten.

An dieser Stelle können Qualitätsmanagementsysteme Standards setzen und den weltwirtschaftlichen Handel erleichtern. Länder und deren Unternehmen können Forderungen ihrer Partner definieren und haben so, zumindest in diesem Bereich, mehr Sicherheit.

Wenngleich auch dadurch Barrieren geschaffen werden, für all die Länder, die sich nicht den Qualitätsstandards anschließen. Doch letztlich überwiegen die Vorteile, was durch die zunehmende internationale Verbreitung von QM-Systemen belegt wird.

\section{Das Unternehmen}

Qualitätsmanagementsysteme zielen ab auf Veränderungen der Verhaltensweisen innerhalb einer auf Qualität und Kundenzufriedenheit ausgerichteten Unternehmenskultur. Das bedeutet, dass die Implementierung eines QM-Systems als Gesamtstrategie für das Unternehmen $\mathrm{zu}$ sehen ist. Die Veränderungen betreffen die komplette Unternehmensorganisation, beginnend bei der Unternehmensleitung. Diese muss nicht nur das System als Ganzes vertreten, sondern gleichzeitig eine Vorbildfunktion übernehmen.

Nur wenn es gelingt diesen Qualitätsgedanken durchgängig im Unternehmen zu etablieren, wird das QM-System als Gesamtstrategie erfolgreich sein. Denn Unternehmen, die die Qualität ihrer Prozesse und Geschäftssysteme erfolgreich strukturieren und verbessern, werden deutlich niedrigere Kosten beim Erreichen ihrer Qualitätsanforderungen an Produkte und / oder Dienstleistungen haben. 


\section{$\underline{\text { Den Mitarbeiter }}$}

Der Mensch ist derjenige, welcher das Bewusstsein der Qualität im Unternehmen hochhält und das Qualitätsmanagementsystem nicht nur mit Leben erfüllt, sondern auch am Leben erhält.

Er hat die Möglichkeit es auszubauen, oder gar zum Scheitern zu bringen. Eine ständige Anschubhilfe seitens der Unternehmensleitung oder anderer Personen, wäre der ungünstigste Fall, der eintreten könnte.

Das Wachhalten des Qualitätsgedankens und ein Qualitätsmanagementsystem, das mit Erfolg praktiziert wird, werden durch eine hohe Motivation der Mitarbeiter begünstigt. Aus- und Weiterbildungsmaßnahmen der Mitarbeiter, ein gutes Betriebsklima, Firmenfeiern etc., sind einige Punkte, die dazu ergriffen werden können.

Die wichtigste Größe in dem Verbund Mensch-Prozess-Qualität, stellt der Mensch selbst dar. Er muss im Stande sein, das Qualitätsmanagementsystem anzuwenden und gleichzeitig in den Prozess eingreifen zu können. Der Mensch kann darüber hinaus auf Probleme des Kunden reagieren und Abhilfemaßnahmen einleiten. Gerade im Umgang mit und bei der Beseitigung von Kundenproblemen, steckt ein großes Zukunftspotenzial für Mitarbeiter [5].

\section{Ergebnis}

Zufriedene Kunden sind die Basis für den Fortbestand jedes Unternehmens. Aus diesem Grunde ist es wichtig Möglichkeiten $\mathrm{zu}$ finden, die die Bestrebungen Kundenzufriedenheit zu erreichen / verbessern unterstützen.

Wie die gemachten Ausführungen belegen, gibt es viele Argumente die in diesem Zusammenhang für die Einführung eines QM-Systems im Unternehmen sprechen.

Auf der Suche nach geeigneten QM-Systemen, findet man beispielsweise die ISO Normenreihe 9000:2000 ff mit ihren Einzelnormen 9000:2000, 9001:2000, 9004:2000 und 19011:2000.

Speziell die ISO Normen 9001:2000 und 9004:2000 fördern dabei die Wahl eines prozessorientierten Ansatzes für die Entwicklung, Verwirklichung und Verbesserung der Wirksamkeit sowie die Effizienz eines Qualitätsmangementsystems, und letztlich dadurch auch die Zufriedenheit der Kunden und der interessierten Parteien durch die Erfüllung ihrer Anforderungen zu erhöhen.

Viele Unternehmen haben sich im Laufe der letzten Jahre dazu entschieden dieses QMSystem einzuführen. Dabei wurden allein von der ISO-Norm 9001:2000 per Dezember 2006 knapp 900.000 Zertifikate - verteilt über 170 Länder - ausgegeben. (Im Vergleich dazu betrug die Anzahl der ausgegebenen ISO 9001:2000 Zertifikate per Dezember 2005 rund 774.000 Stück - verteilt auf 161 Länder.) [6].

Nur wer konsequent an der Verbesserung seiner Produkt- und / oder Dienstleistungsqualität sowie der Steigerung seiner Kundenzufriedenheit arbeitet wird langfristig am Markt bestehen können - QM-Systeme unterstützen diese Bestrebungen. 


\section{Literaturverzeichnis und andere Quellen}

[1] KAMISKE, G. F.; BRAUER, J.-P.: Qualitätsmanagement von A bis Z, Carl Hanser Verlag München Wien 2006, S. 210, ISBN 3-446-40284-5

[2] QZ Qualität und Zuverlässigkeit: Qualität und Kunden entscheiden über Geschäftserfolg, in QZ Jahrgang 53, Nr. 2, Carl Hanser Verlag, München 2008, S. 76

[3] KETTING, M.: Geschichte des Qualitätsmanagements, in Masing, W. (Hrsg.), Handbuch Qualitätsmanagement, Carl Hanser Verlag München Wien 1999, S. 26 ff, ISBN 3-446-19397-9

[4] SEGHEZZI, H. D.: Integriertes Qualitätsmanagement, Carl Hanser Verlag München Wien 2003, S. 180, ISBN 3-446-22005-4

[5] WACK, PETER: QM im Land des Lächelns, QZ Jahrgang 46, Nr. 3, Carl Hanser Verlag München 2001, S. $280 \mathrm{ff}$

[6] ISO: The ISO Survey 2006, in ISO News and media - News - 2007, Ref.: 1089, 23.11.2007, http://www.iso.org/iso/pressrelease.htm?refid=Ref1089

\section{Grantová podpora}

Článok vznikol na základe podpory projektov medzinárodnej vedecko-technickej spolupráce Nem/SR/ZU1/07- MVTS1 - Core business v poštovom podniku a projektu medzinárodnej vedecko-technickej spolupráca Nem/SR/ZU2/07-MVTS2 Zákaznícka spokojnost' $\mathrm{v}$ bankovom sektore. 